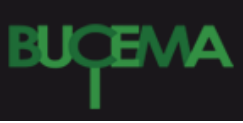

Bulletin du centre d'études médiévales d'Auxerre | BUCEMA

Hors-série $n^{\circ} 9$ | 2016

Géolocalisation et sources anciennes?

\title{
Géolocaliser des cartes anciennes : procédure
}

Claire Audelan, Marion Humbert, Clémence Lescuyer, Constance de Vergnette de la Motte et sous la direction d'Alain Guerreau

\section{(2) OpenEdition}

\section{Journals}

Édition électronique

URL : https://journals.openedition.org/cem/14148

DOI : $10.4000 /$ cem. 14148

ISSN : 1954-3093

Éditeur

Centre d'études médiévales Saint-Germain d'Auxerre

\section{Référence électronique}

Claire Audelan, Marion Humbert, Clémence Lescuyer, Constance de Vergnette de la Motte et sous la direction d'Alain Guerreau, «Géolocaliser des cartes anciennes : procédure », Bulletin du centre d'études médiévales d'Auxerre / BUCEMA [En ligne], Hors-série n 9 | 2016, mis en ligne le 27 novembre 2015, consulté le 05 mars 2023. URL : http://journals.openedition.org/cem/14148 ; DOI : https://doi.org/ $10.4000 /$ cem. 14148

Creative Commons - Attribution - Pas d'Utilisation Commerciale - Partage dans les Mêmes Conditions 4.0 International - CC BY-NC-SA 4.0

https://creativecommons.org/licenses/by-nc-sa/4.0/ 


\section{Géolocaliser \\ des cartes anciennes: procédure}

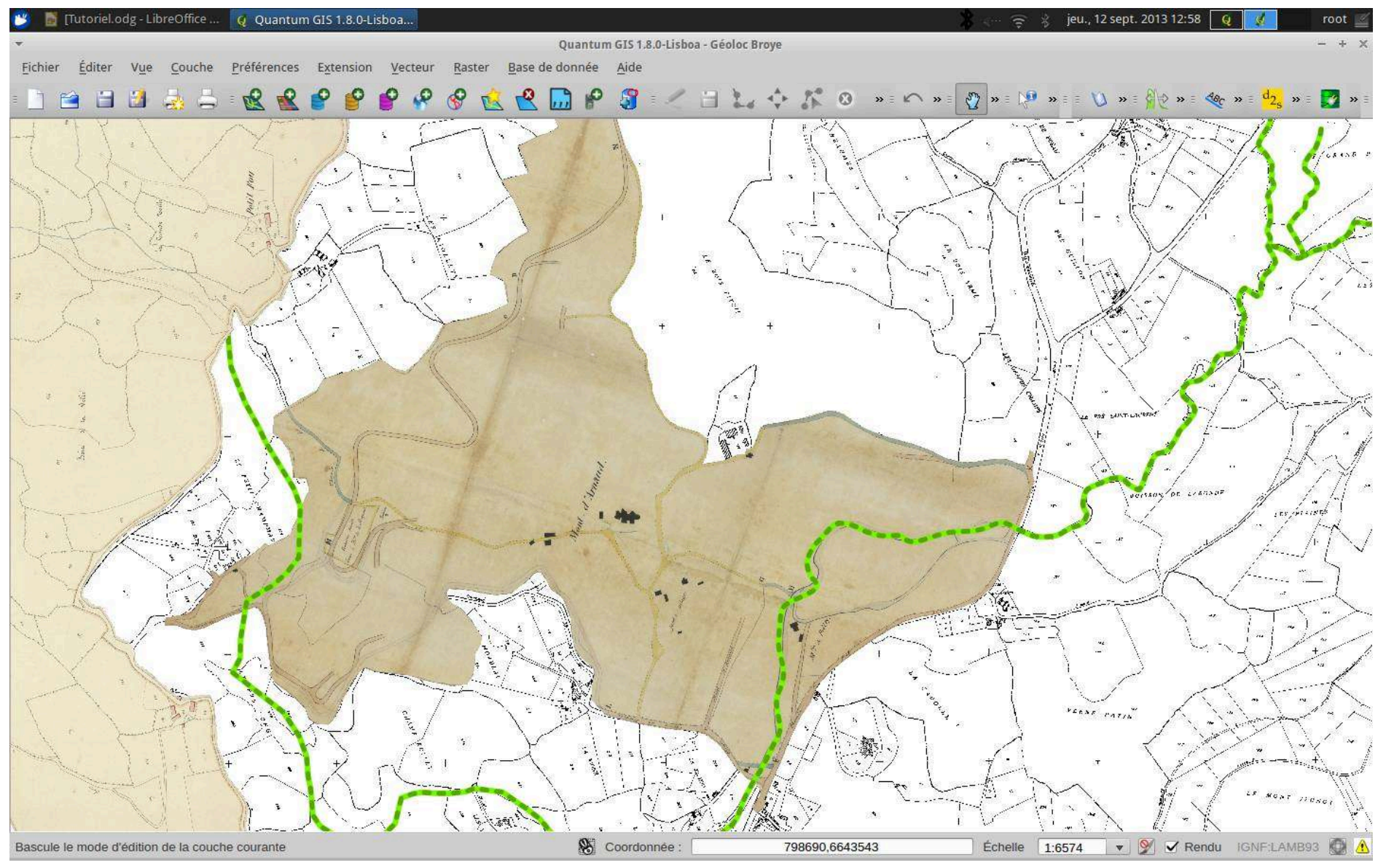

Claire AUDELAN

Marion HUMBERT

Clémence LESCUYER

Constance DE VERGNETTE DE LA MOTTE

Sous la direction d'Alain Guerreau 
La géolocalisation de documents cartographiques (cartes, plans, photographies aériennes) est une opération facile à réaliser à l'aide des outils actuels ; les manipulations de documents géolocalisés permettent d'extraire de ces objets une multitude d'informations inaccessibles par une lecture directe, il s'agit désormais d'une étape indispensable de toute étude historique faisant appel à ce type de sources ; le présent papier a pour objet d'expliquer comment effectuer cette opération. [NB la procédure décrite vise les documents anciens, mais s'applique de la même manière à tout type de document cartographique].

\section{Les objets}

\section{1. qu'est-ce qu'une carte géolocalisée?}

Une carte géolocalisée est un fichier informatique représentant une image d'une portion de la surface terrestre munie coordonnées géographiques précises ; si l'on ouvre ce fichier à l'aide d'un logiciel d'imagerie ordinaire, on obtient une image non moins ordinaire ; si on l'ouvre dans un système d'information géographique (SIG), l'image est disposée dans un système de coordonnées de référence, de telle sorte que deux images correspondant à la même zone seront superposées, ou que deux images correspondant à des zones contiguës seront jointives. Dans une carte géolocalisée, chaque élément de base (point ou pixel) est repéré par ses coordonnées $\mathrm{X}$ et Y. On peut donc parler d'un fichier image muni de « métadonnées » géographiques.

觢 trois termes sont en concurrence, entre lesquels l'usage n'a pas encore tranché : géolocalisation, géoréférencement et géocodage ; pour le moment, ils sont à peu près synonymes.

\section{2. pourquoi des cartes géolocalisées?}

Le principe de fonctionnement d'un SIG est l'empilement de « couches » : chaque fichier ouvert correspond à une couche précise. On peut modifier l'ordre d'empilement, et surtout modifier la transparence de telle ou telle couche, de telle sorte que l'on peut aisément et très exactement comparer deux couches superposées ; on conçoit immédiatement l'intérêt d'une telle possibilité pour toute comparaison de cartes correspondant à deux dates différentes (qu'il s'agisse aussi bien de bâtiments que de parcellaires, de réseau viaire, de type d'utilisation du sol, etc). Mais l'intérêt n'est pas moindre dans la synchronie, puisque l'on peut ainsi « monter » dans une seule image un ensemble de documents séparés, par exemple des jeux de photos aériennes ou des sections de terrier ou de plan cadastral. On doit aussi souligner que le système de coordonnées est le plus souvent un carroyage métrique ou kilométrique, ce qui facilite une série de calculs de distances et de surfaces. Enfin, s'agissant de cartes anciennes plus ou moins inexactes géométriquement, il faut savoir qu'une géolocalisation soignée permet une rectification complète : la carte est modifiée localement de manière à rétablir une exacte proportionnalité avec le terrain.

\section{3. les principaux types de documents concernés.}

Toute représentation d'une portion de la surface terrestre peut être utilement géolocalisée. Ce qui signifie que toutes les échelles sont utilisables ; la représentation peut être celle d'une zone de fouille de 10 mètres sur 10, aussi bien que celle d'un continent. L'historien se tourne naturellement d'abord vers des documents anciens : portulans, tibériades, cartes anciennes, plans-terriers, plans cadastraux, plans de forteresses, plans d'urbanisme et ainsi de suite. Mais les documents plus récents sont tout autant à prendre en compte, en particulier tous les jeux successifs de photos aériennes, les plans de bâtiments, les plans de fouille, ainsi que toutes les cartes actuelles. On ne doit pas non plus négliger toutes les cartes des atlas historiques : celles-ci sont souvent disjointes, représentées à des échelles différentes, l'insertion dans un SIG après géolocalisation permet d'avoir une vue homogène, période par période. On pourra se rendre compte concrètement de l'intérêt de ce genre d'outil en consultant attentivement un des meilleurs SIG en ligne, le Géoportail (www.geoportail.fr). Des SIG totalement ou en partie historiques apparaissent de toutes parts. (Par exemple : http://cultures.toulouse.fr/urban-hist ou http://www.archives71.fr/arkotheque/client/ad_saone_et_loire/plans_terriers/plans_terriers.php).

Lorsque les fichiers informatiques ne sont pas déjà disponibles, on utilisera de préférence le scanner si le format le permet ; si l'on doit employer un appareil photographique, on doit d'abord se livrer à un petit calcul : comment obtenir une définition d'au moins 150 dpi (pixel per inch, soit 1120 pixels pour $10 \mathrm{~cm}$ ) ; ce qui, pour donner un ordre de grandeur, permet de numériser une image de 41x55 cm avec un appareil qui enregistre 8 millions de pixels, 56x75 cm avec un appareil qui enregistre 15 millions de pixels. Pour numériser une image plus grande, on photographie par parties et on rassemble les morceaux (avec un logiciel de « stitching » comme hugin) ou on les traite séparément. 


\section{Outils et matériaux}

\section{1. les logiciels.}

La totalité des opérations ne nécessite que deux logiciels : un logiciel de traitement d'images, et un SIG disposant d'un outil de géolocalisation. On ne saurait trop recommander l'usage de logiciels libres ; en l'occurrence Gimp comme traitement d'images et QGIS comme SIG. Bien entendu le mieux est de travailler dans un environnement libre de type linux, mais ces deux logiciels sont disponibles pour toutes les plates-formes.

A l'automne 2013, les dernières versions sont Gimp 2.8.6 et QGIS 2.0. Ces deux logiciels sont puissants et efficaces, ils supportent avantageusement la comparaison avec des outils propriétaires qu'il est onéreux de se procurer et onéreux de tenir à jour.

Dans tous les cas, il faut savoir que les fichiers-images représentant des cartes sont des fichiers volumineux, voire gigantesques. Les traitements sont donc longs et requièrent de la place en mémoire vive : il vaut mieux travailler avec une machine puissante et disposant de beaucoup de RAM (compter au moins 8 go, plus si possible).

[Gimp et QGIS dsisposent de manuels bien faits ; on trouve de nombreux tutoriels ici ou là].

\section{2. les sources du géoréférencement.}

La procédure de géolocalisation consiste en gros à indiquer les coordonnées exactes d'un nombre suffisant de points sur l'image que l'on traite ; il faut donc connaître ces coordonnées d'une manière ou d'une autre. Pour cela, il faut disposer d'une carte représentant la même zone et déjà géolocalisée, de manière à pouvoir opérer autant de reports que nécessaire ; le plus efficace est d'avoir ces fichiers sur sa propre machine ; sinon, on peut utiliser tel ou tel outil en ligne qui permet de connaître, pour tel ou tel point, les coordonnées exactes, mais dans ce cas-là, on devra reporter ces coordonnées manuellement, ce qui est bien plus long et fastidieux.

Le mouvement de l'open data a d'ores et déjà incité diverses institutions détentrices de telles cartes à les proposer au téléchargement libre.

En France, l'Institut Géographique National (IGN) constitue la source principale ; voir : http://professionnels.ign.fr/catalogue. De très nombreuses cartes sont en accès libre ; depuis 2010, l'IGN accorde l'accès libre aux institutions de recherche et d'enseignement pour la plupart de ses cartes ; l'accès est accordé aux institutions qui en font la demande. Dans ce cadre, on dispose d'une base qui suffit à la quasi totalité des besoins s'agissant de zones de l'hexagone ou des dom-tom.

A l'échelle européenne, l'offre est moins abondante, mais commence à s'étoffer. La ressource la plus intéressante est le fichier EuroGlobalMap, qui fournit d'excellentes cartes vectorielles de l'Europe, en particulier le réseau hydrographique, les routes, les circonscriptions administratives (http://www.eurogeographics.org/form/topographic-data-eurogeographics). L'Institut de statistiques européennes (eurostat) fournit également les circonscriptions, mais aussi le centroïde de toutes les communes (http://epp.eurostat.ec.europa.eu/portal/page/portal/gisco_Geographical_information_maps/popups/references/admin istrative_units_statistical_units_1) ; l'Agence européenne de l'environnement (eea) fournit un excellent fichier DEM (altitudes) et un fichier de l'utilisation des sols (Corine) : http://www.eea.europa.eu/data-and-maps\#tab-datasets.

\section{3. choix d'une précision et d'un système de coordonnées de référence (SCR).}

Avant de se lancer dans les opérations, il faut réfléchir à la précision et au SCR. La précision dépend avant tout de l'échelle choisie, donc de la taille de l'objet représenté. A petite échelle (type 1/1000000), l'Europe ou l'hexagone, une précision de l'ordre de $10 \mathrm{~km}$ est suffisante ; à moyenne échelle (1/500000 à 1/80000), une région ou un département, le kilomètre suffit ; à grande échelle (plus de 1/50000), il faut une précision métrique ou meilleure. En France, on choisira de préférence le SCR dit « Lambert93 » (EPSG:2154), qui est le SCR officiel, et celui employé par l'IGN ; pour l'Europe, on préférera le système ETRS89-LCC (EPSG:3034) ou ETRS89LAEA (EPSG:3035), qui donnent des cartes à l'allure habituelle et qui sont préconisés par tous les organismes européens.

[Sur toutes les questions techniques de géodésie, de projections, de système de coordonnées, etc, consulter les pages correspondantes de fr.wikipedia.org]. 


\section{Préparer la carte : Gimp}

La plupart des cartes anciennes nécessitent une étape préalable à la géolocalisation proprement dite.

\section{1. identifier}

Il faut commencer par savoir précisément ce que représente la carte, ce qui est souvent difficile ; si la zone représentée est de taille modeste, que le plan n'est pas orienté, et qu'il s'agit d'une pièce d'archive plus ou moins isolée, cela peut demander du temps ; on utilise surtout les toponymes (s'il y en a ...), et l'on essaye de découvrir dans la région que l'on pense être la bonne un emplacement présentant les mêmes caractères.

\section{2. corriger et orienter}

Cela fait, il faut, si besoin est, réorienter l'image et la détourer.

On travaille à l'aide de l'éditeur d'image GIMP. « Fichier » « Ouvrir » pour afficher l'image. Il faut d'abord procéder à toutes les améliorations nécessaires (luminosité-contraste, couleur, netteté, etc), car ces modifications ne sont plus possibles après la géolocalisation (elles la font disparaître). La deuxième étape consiste à réorienter (à peu près) la carte vers le nord si elle ne l'est pas d'origine.

Pour éviter que la rotation ne rogne l'image, on crée une image vide de taille double : " Fichier » « Nouvelle image ». Dans la rubrique « Taille d'image », on double le nombre de pixels en largeur et en hauteur puis on valide.

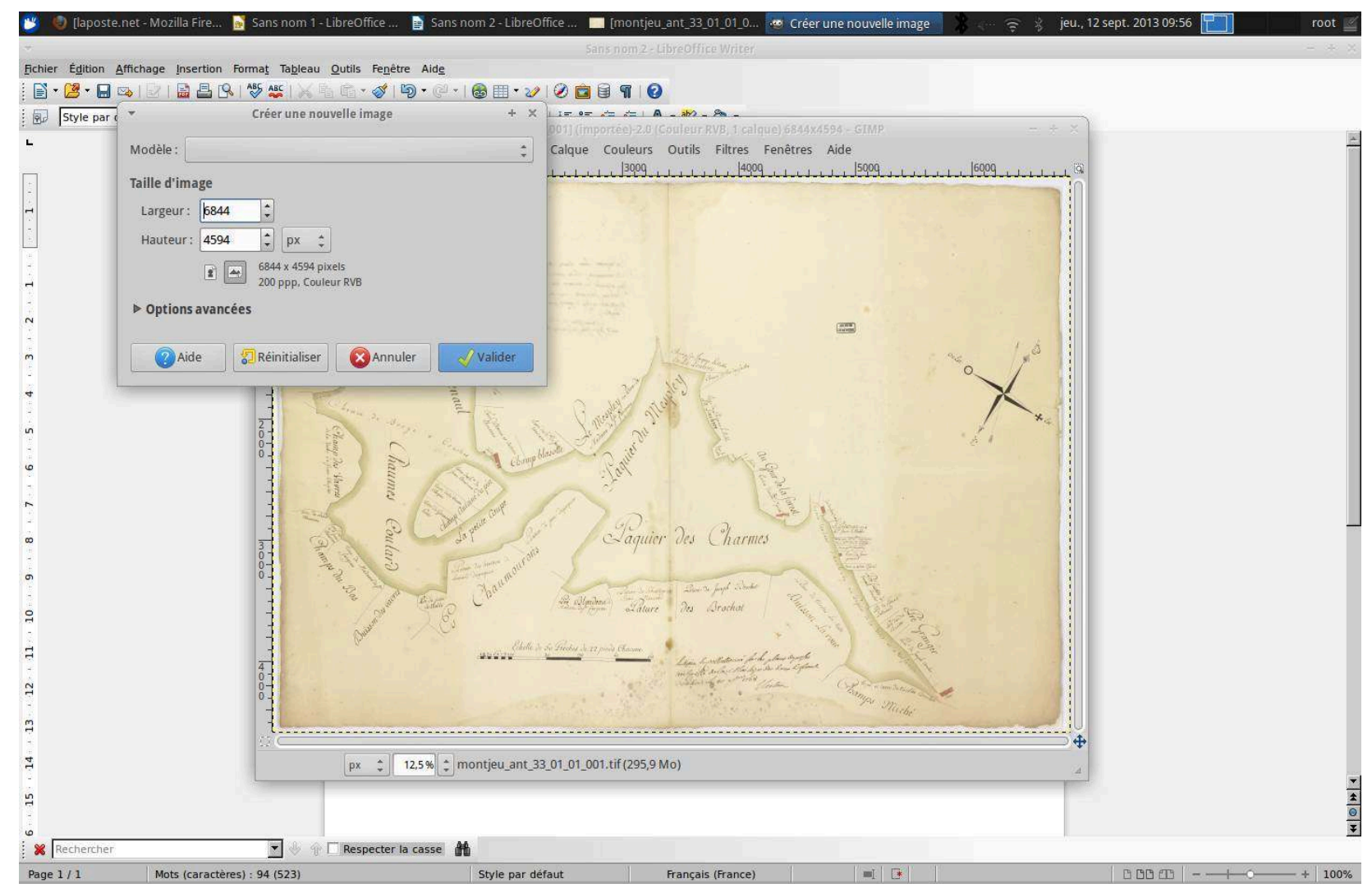

Une fois cette nouvelle image (vide) créée, revenir sur la carte puis « Edition » « Copier ». « Edition » « Coller » sur le rectangle blanc qui vient d'être créé. L'image s'affiche normalement en plein milieu. « Outils » puis « Outils de transformation », « Rotation ». On peut ensuite soit directement entrer un angle de rotation, soit faire tourner l'image à l'aide du curseur. Clic sur « Rotation ». 


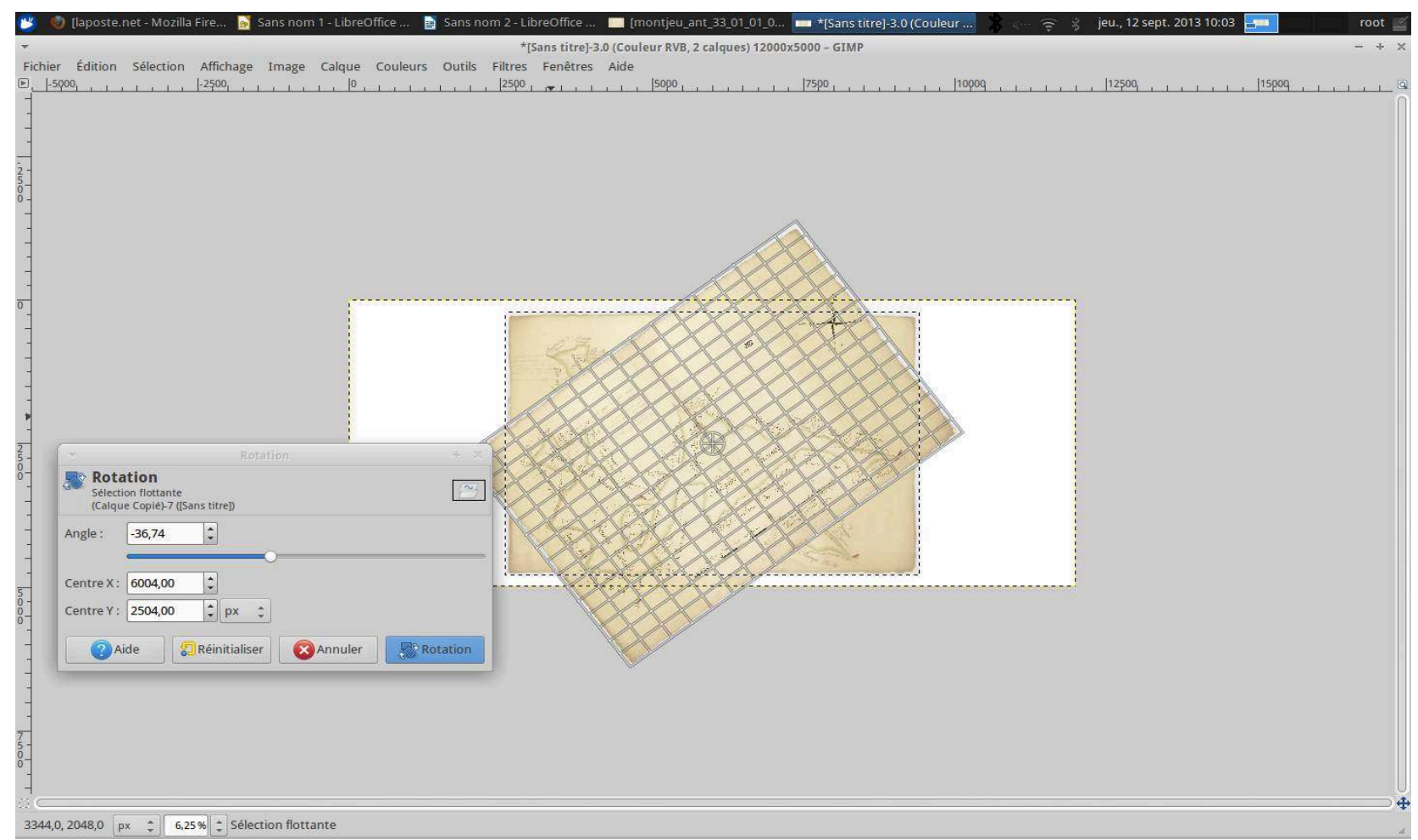

\section{3. détourer}

Une fois la carte réorientée (le nord en haut), il est nécessaire d'en enlever tout le superflu nongéolocalisable (partie blanche, rose des vents, échelle, notes etc.). Pour ce faire, " Outils », " Chemins ». Faire ensuite le tour de la carte en positionnant des points régulièrement. Cette étape est TRÈS importante car elle détermine la forme de la carte telle qu'elle apparaîtra une fois géolocalisée.

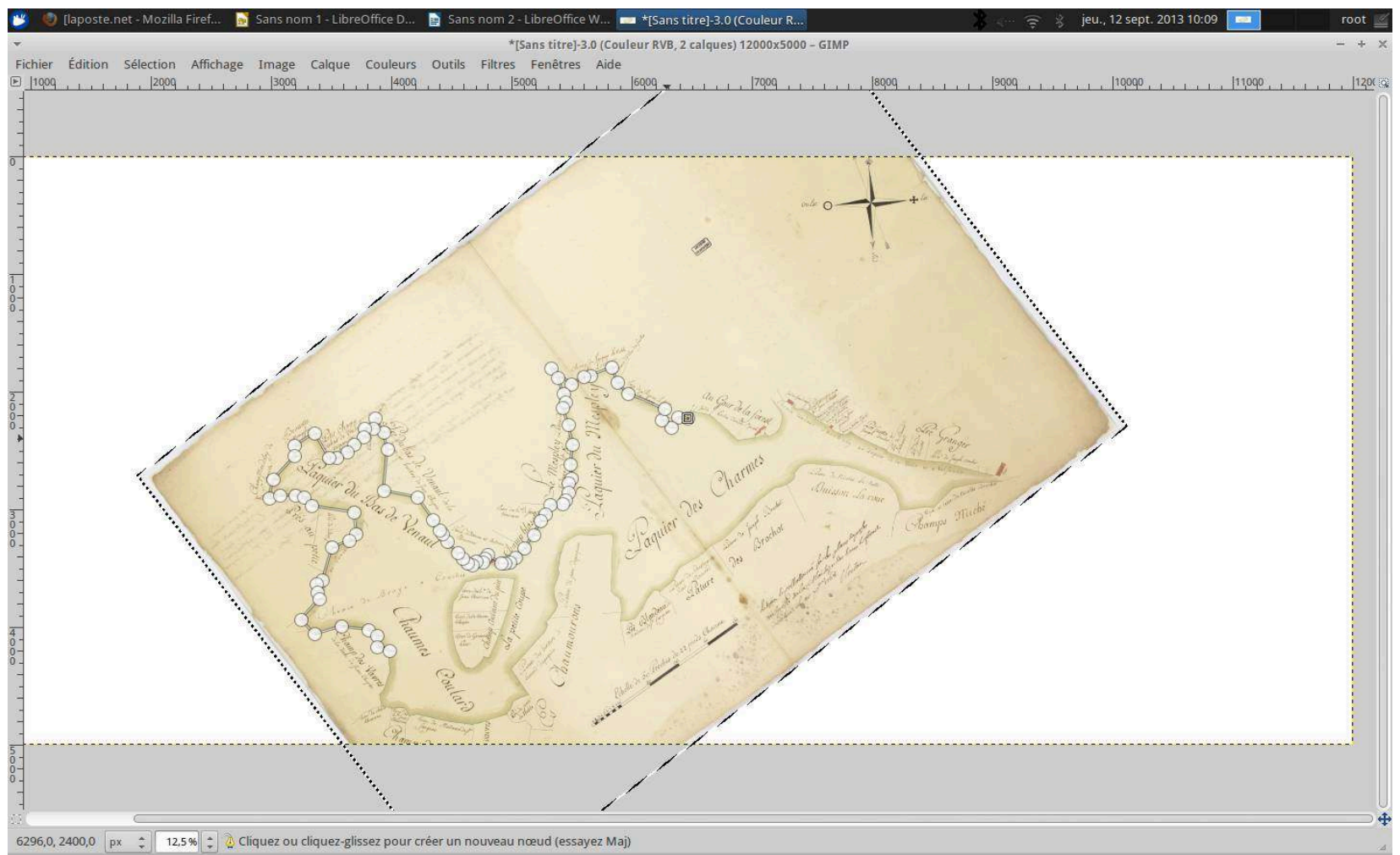

Pour clore le chemin, CTRL-clic sur le premier point quand on le rejoint. « Sélection » « Depuis le chemin ». Le fond de l'image doit normalement disparaître et des pointillés se matérialiser autour de la carte. « Edition », « Copier » puis « Fichier », « Créer », « Depuis le presse-papier ». La carte apparaît 
normalement sur un fond en damier (le damier indique qu'il s'agit d'une zone transparente). « Fichier », « Exporter ». Si nécessaire, Il faut à ce moment de changer le format du fichier pour le format TIFF, qui est le seul format d'image raster facilement géoréférençable. Pour le choix de la compression : « LZW ». Une fois l'image exportée, fermer GIMP, sans enregistrer les transformations.

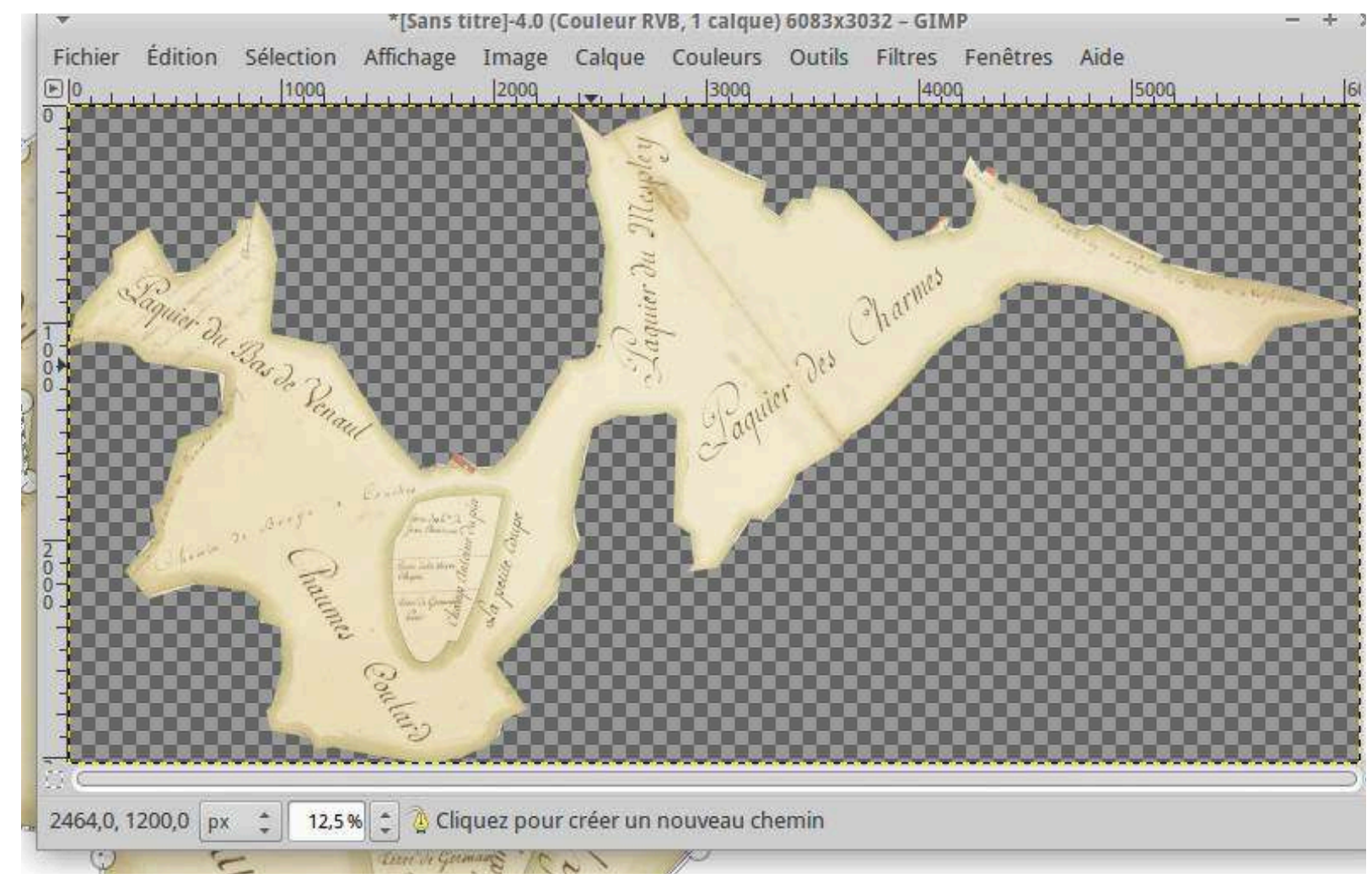

L'image est prête pour la géolocalisation. 


\section{Installer une carte de référence :QGIS-1}

Avant de procéder à la géolocalisation d'une carte ancienne, il est nécessaire de mettre en place, dans Qgis, une couche déjà géolocalisée permettant le report des coordonnées à partir de cette carte.

Le choix de cette carte dépend de l'emplacement et de la taille de la carte à géolocaliser ; pour une carte à grande échelle, les fichiers du cadastre géolocalisé diffusés par l'IGN constituent un des meilleurs choix. Cette carte de référence doit être affichée en tant que couche raster à l'aide de la rubrique « Couche » dans le menu puis « Ajouter une couche raster » dans le menu déroulant.

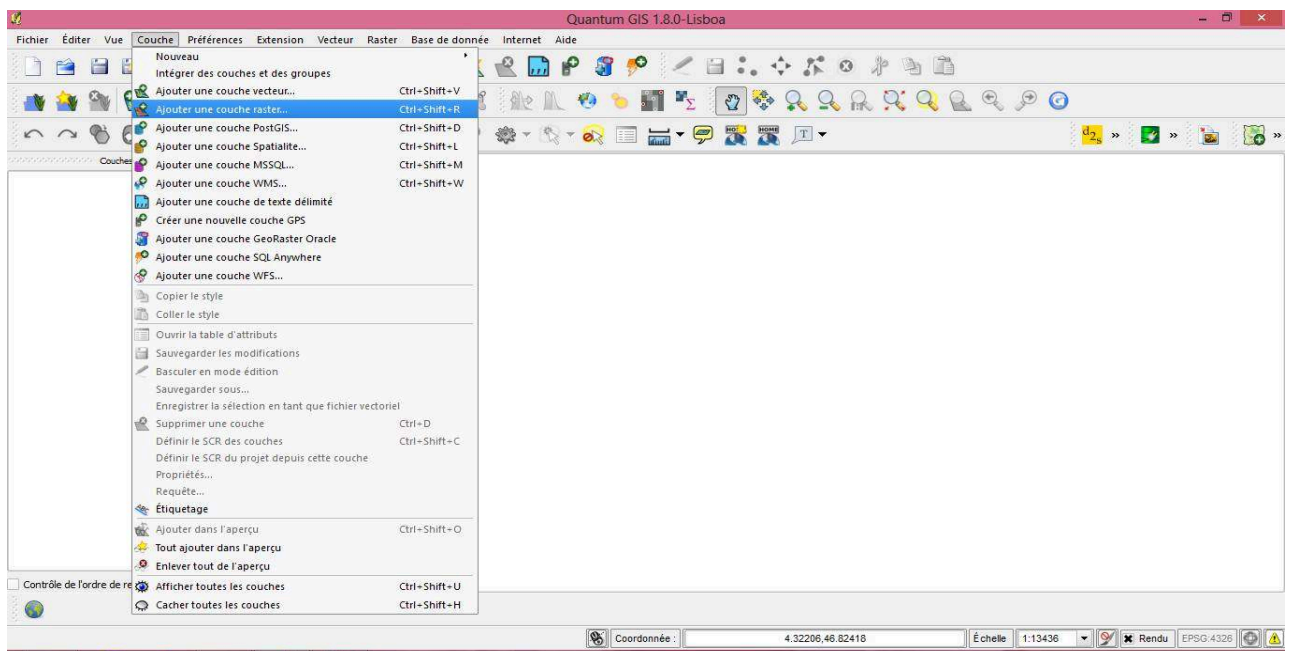

Une fois la couche voulue sélectionnée, une fenêtre s'affiche pour choisir le système de coordonnées de référence. En France, on utilise le SCR « Lambert93 » (EPSG:2154).

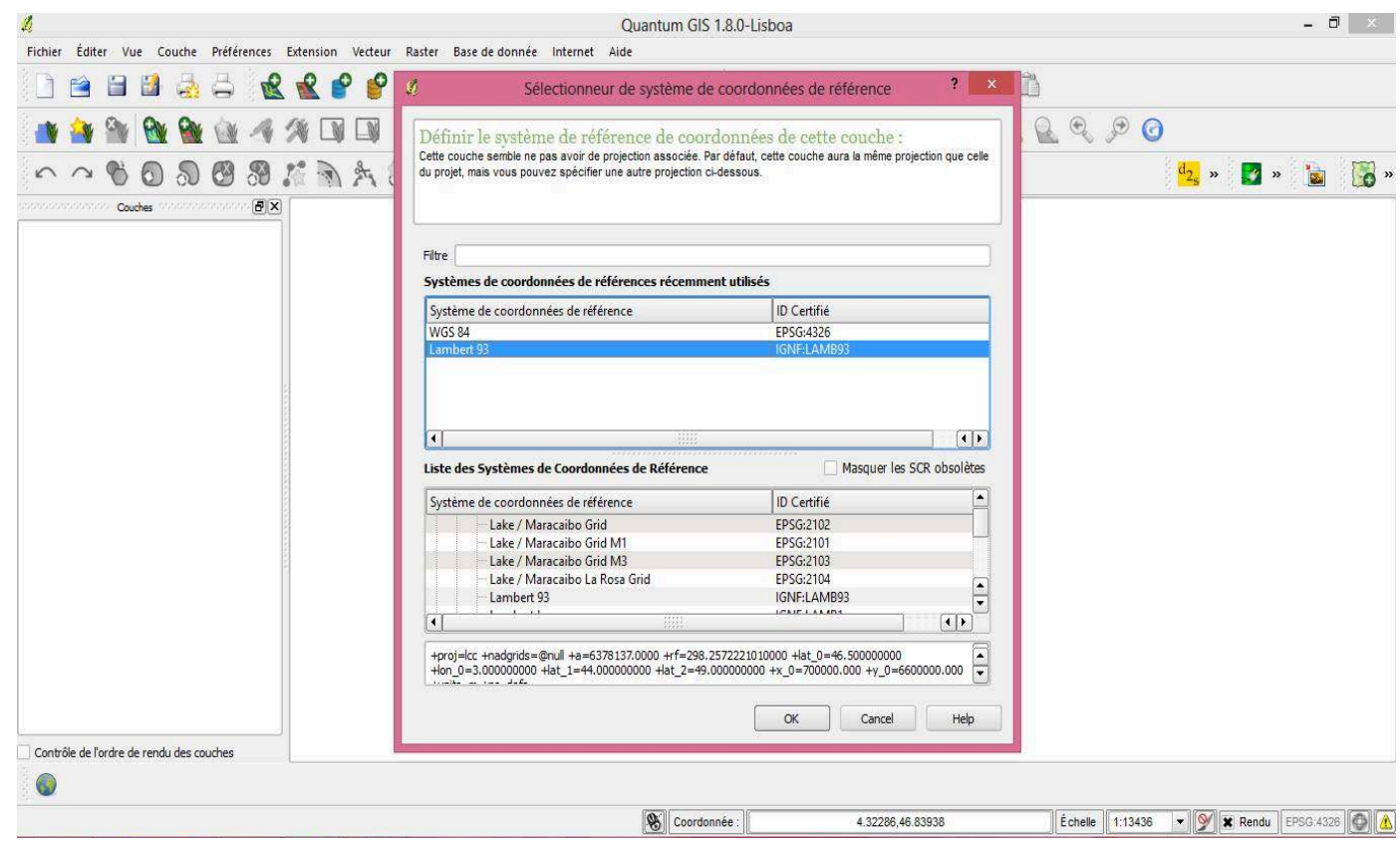

Si tout fonctionne bien, la couche s'affiche dans la fenêtre principale et sa présence est signalée dans une fenêtre à gauche de l'écran. Si cette couche de référence est constituée de plusieurs fichiers, on procède de la même manière pour les fichiers suivants. 


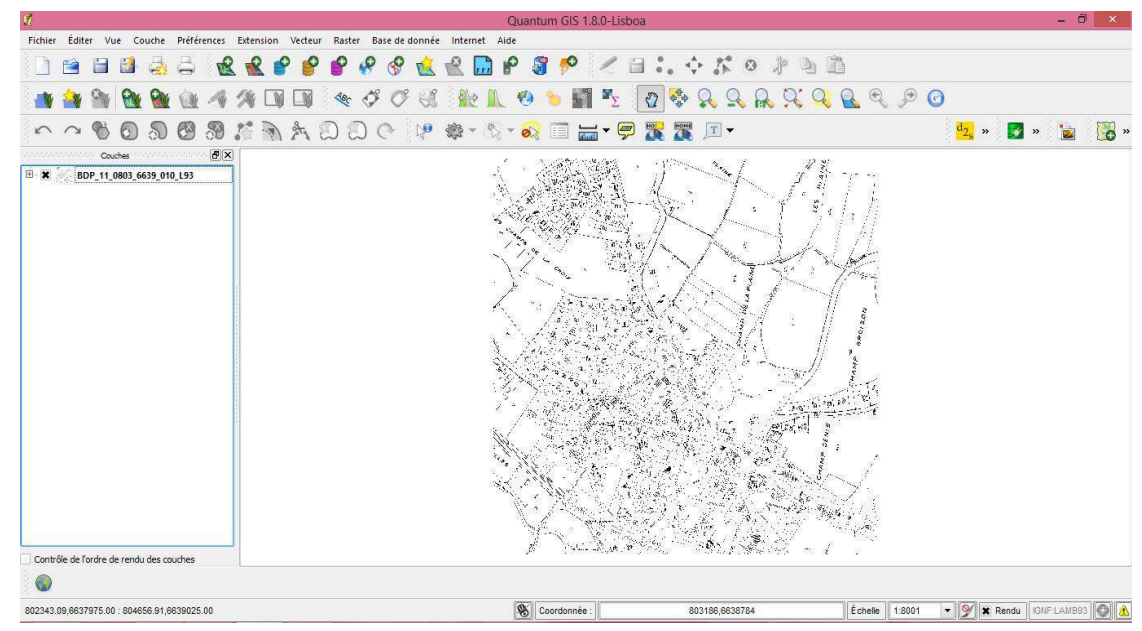

Si l'on souhaite afficher davantage d'informations sur cette couche déjà géolocalisée et servant de support de base, on peut, par exemple, utiliser des données supplémentaires provenant de l'IGN : le réseau routier, hydrographique ou tout simplement les limites des communes. Dans le menu déroulant, il faut choisir « Ajouter une couche vecteur ».

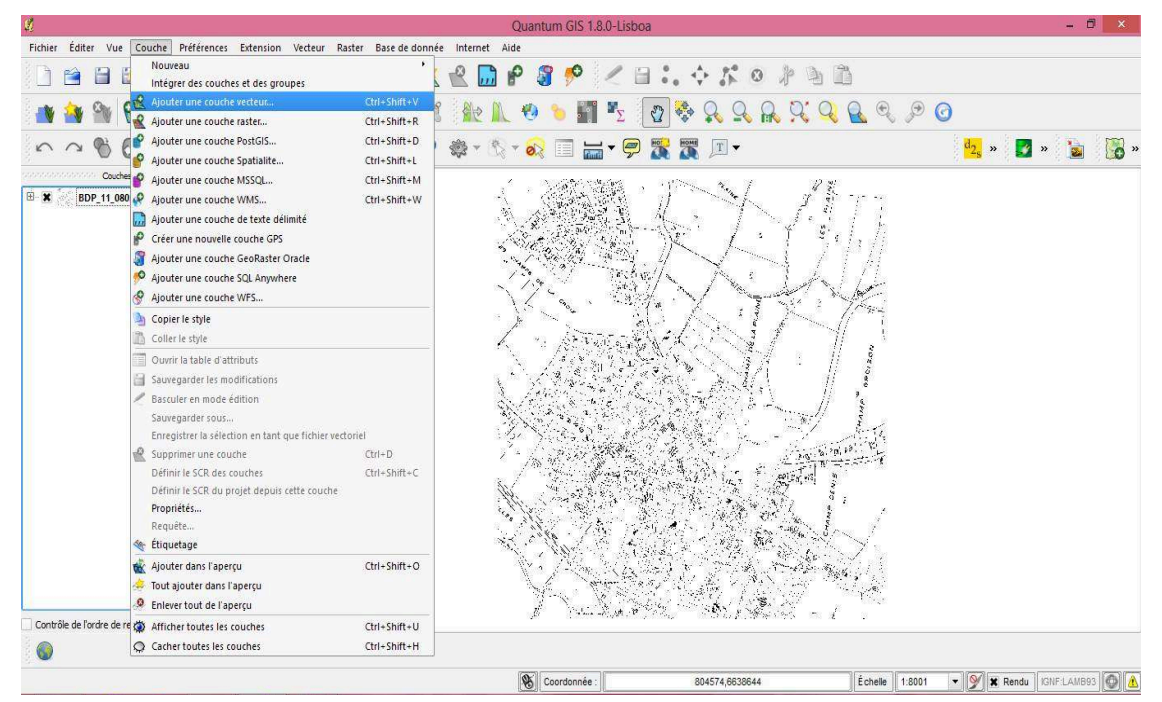

Puis, sélectionner la couche souhaitée dans le cadre « Source » et « Jeu de données ».

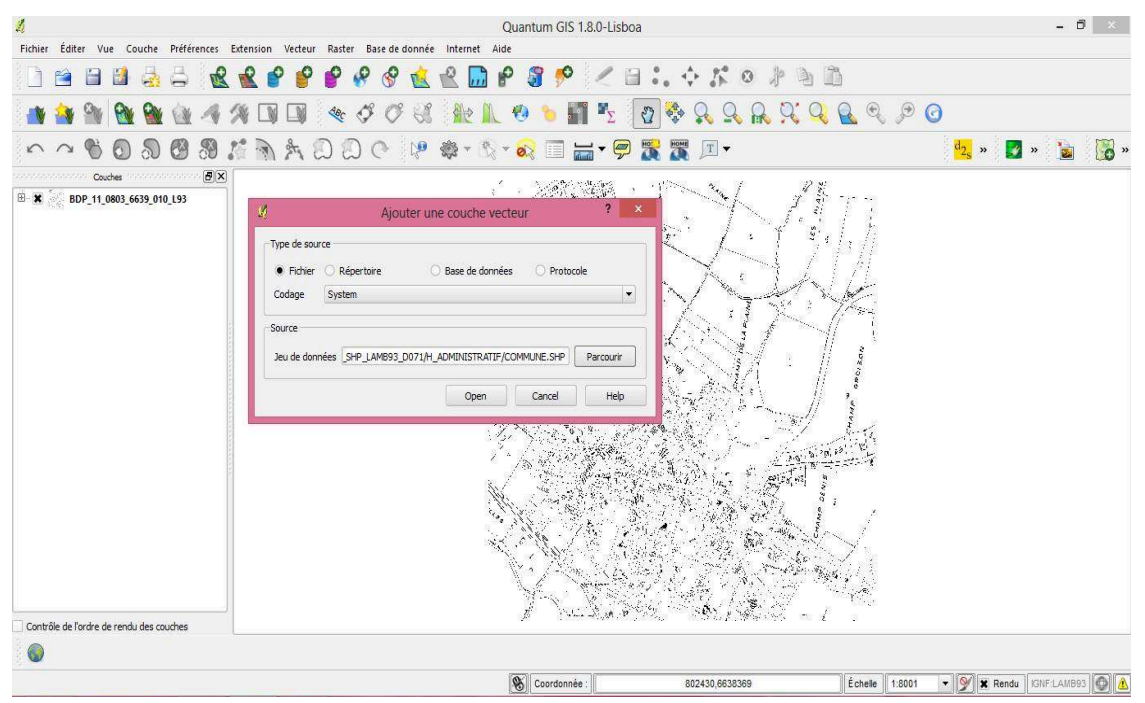


Si l'affichage ne correspond pas à ce que l'on souhaite, faire un un clic droit sur la couche à modifier (dans la fenêtre de gauche .

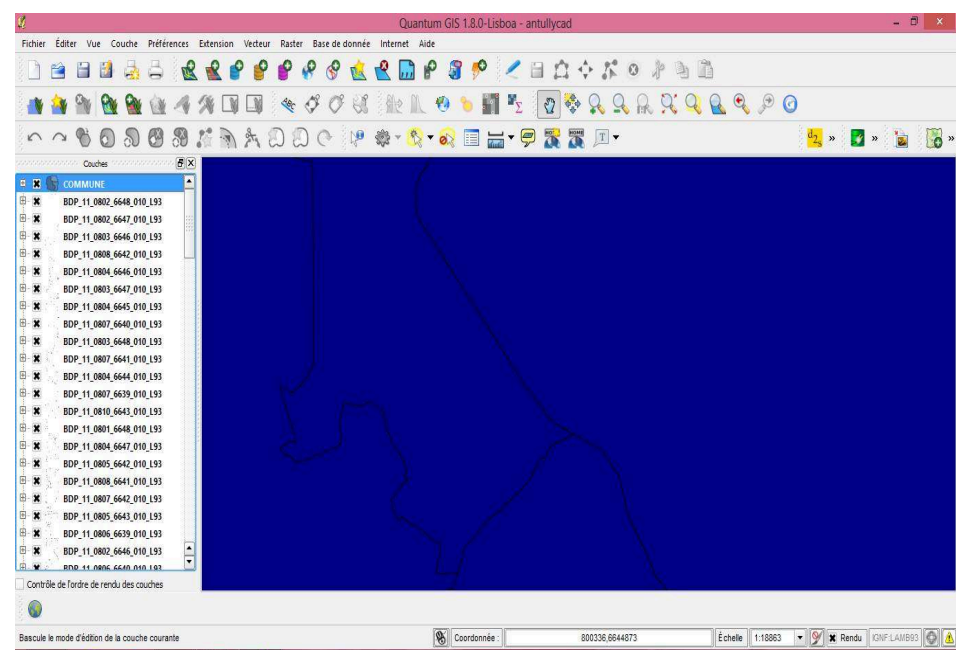

Dans la fenêtre qui apparaît, choisir le style puis l'encadré intitulé « Modification ». Les propriétés du symbole s 'affichent et c'est le « Style de remplissage » qu'il faut changer, en passant de « Continu » à « Pas de brosse ».
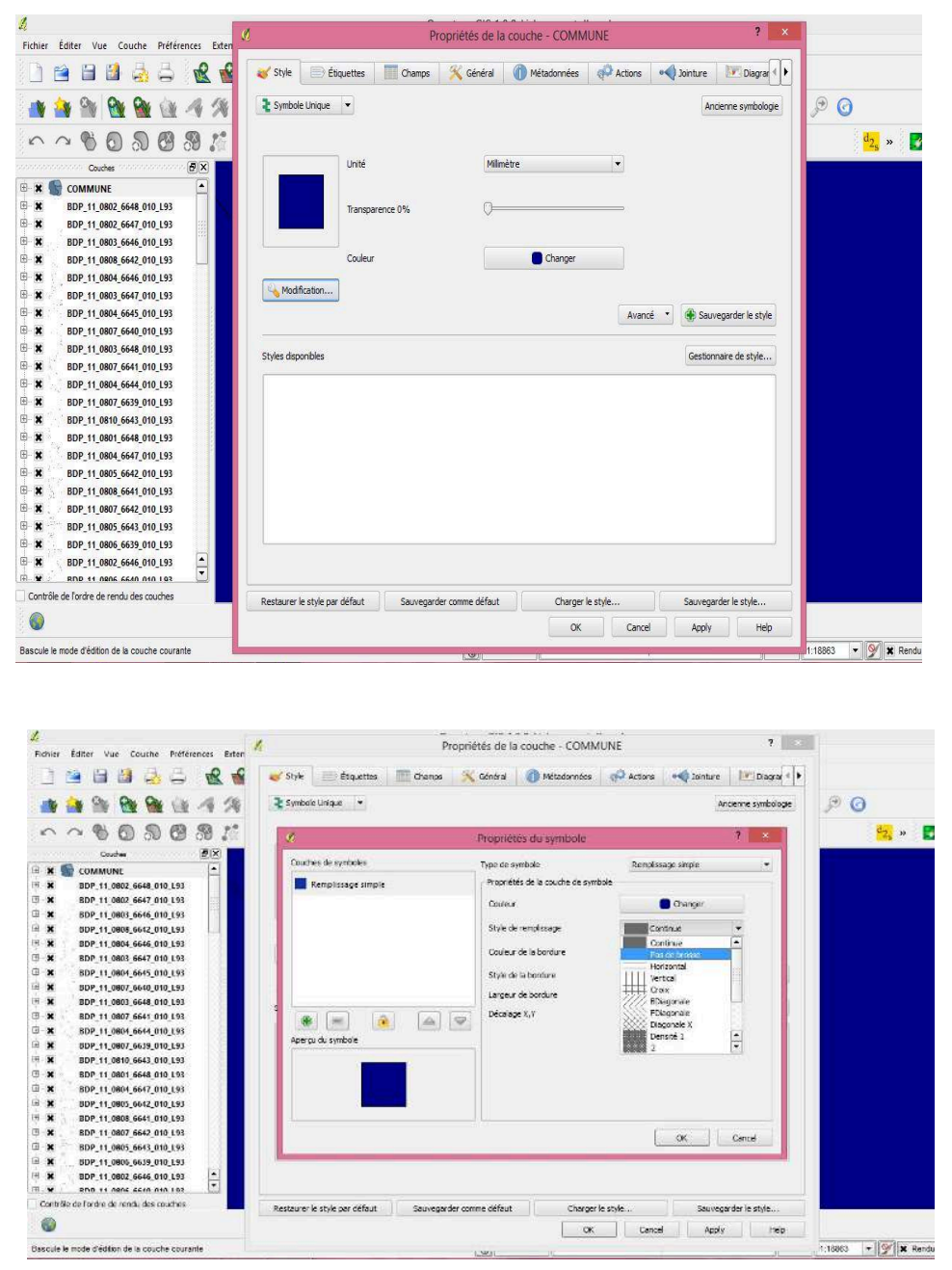

Après l'application de ce changement, les couches sont lisibles en totalité. 


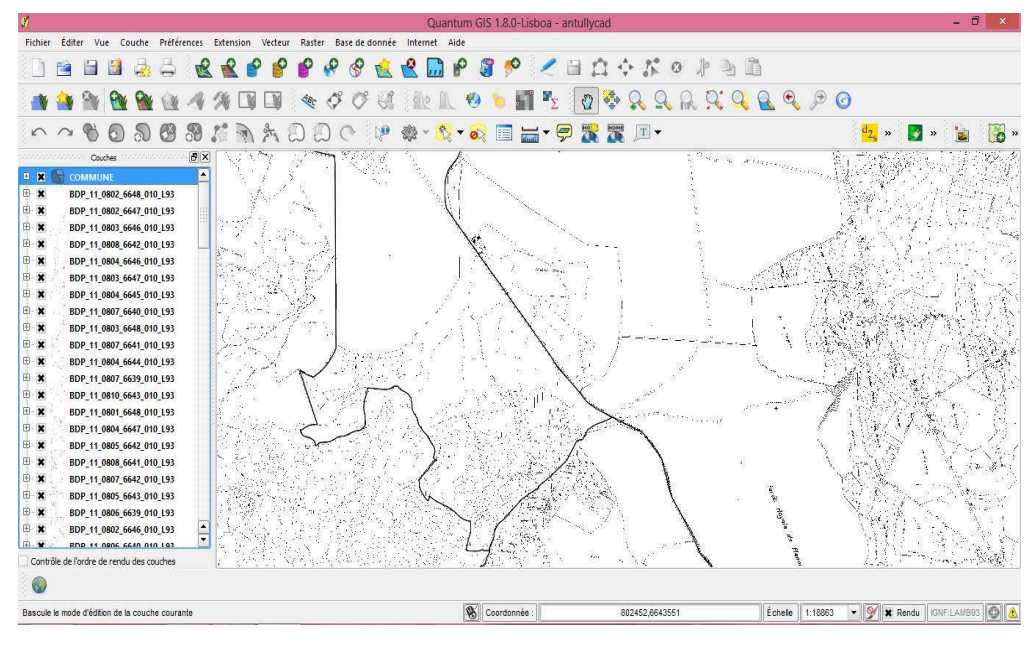

Si l'on veut que s'affichent des informations écrites sur les couches comme le nom des communes, il s'agit également de modifications de propriétés mais à partir de l'onglet « Etiquette » et en demandant d ' « Afficher les étiquettes ».

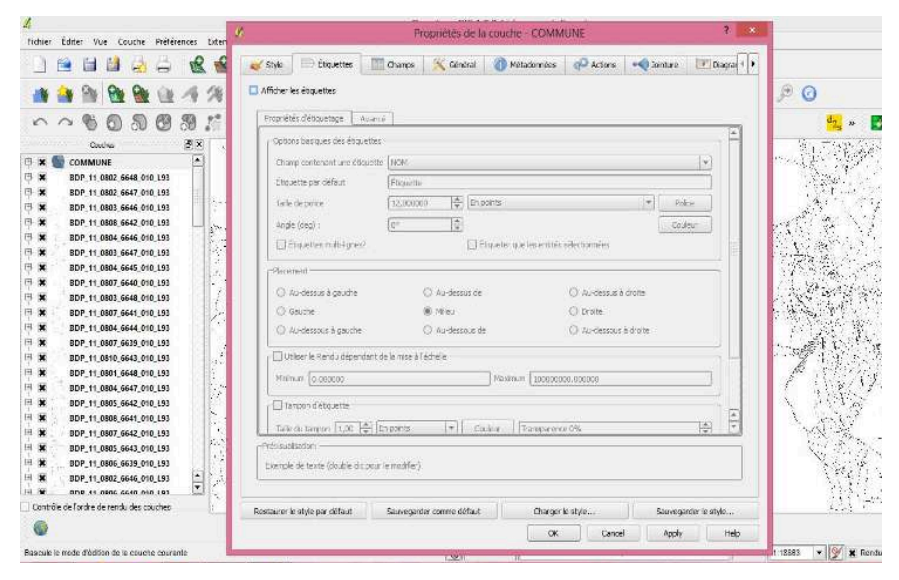

Il faut alors sélectionner l'information que l'on souhaite afficher sur la couche dans l'onglet « Propriétés d'étiquetage » puis « Options basiques des étiquettes » puis « Champ contenant une étiquette ». A cet endroit, toutes les propriétés des étiquettes peuvent être modifiées.
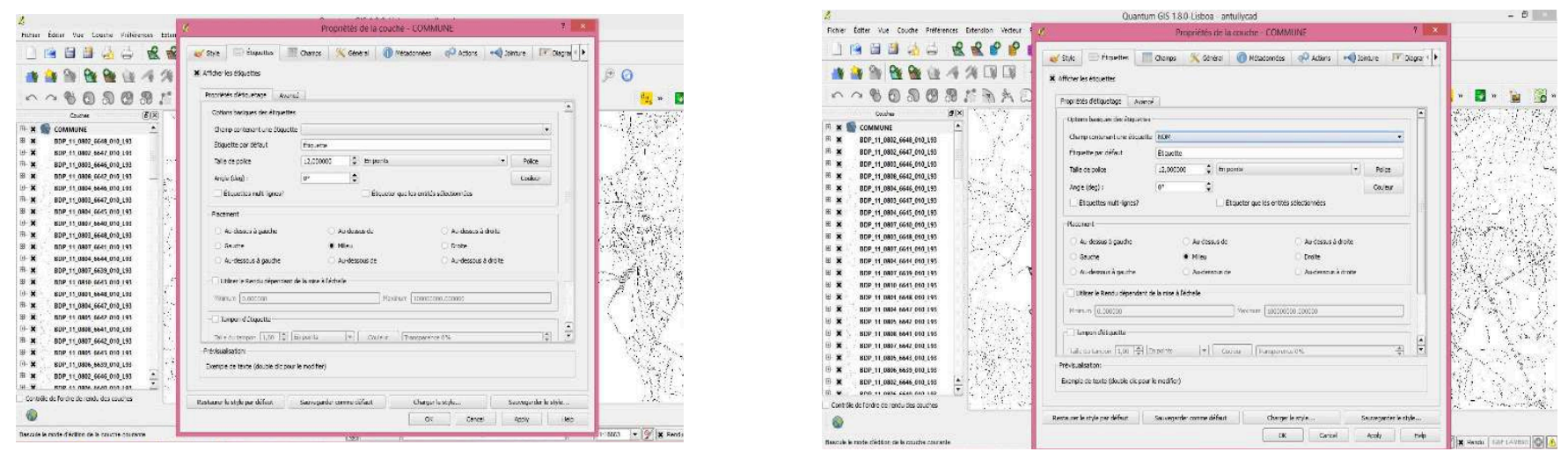

L'affichage des diverses couches vecteurs fonctionne selon le même système.

Voici le résultat : 


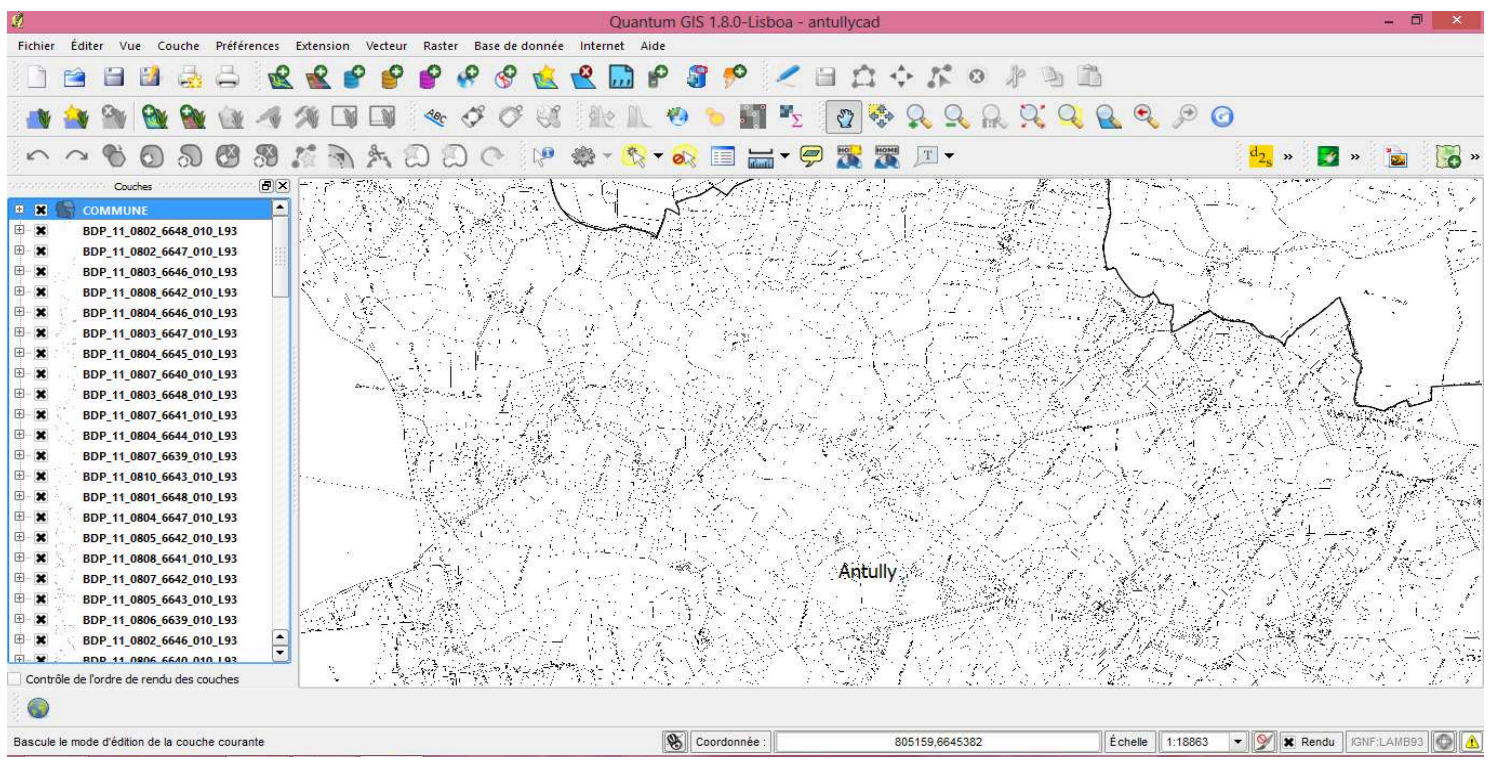




\section{Géolocaliser la carte ancienne : QGIS-2}

Deux situations se présentent :

soit on dispose d'un fond géolocalisé, par exemple des cartes de l'IGN (couches raster et vectorielle) $=>$ cf. 1

soit on a seulement accès à un site montrant des cartes géolocalisées, par exemple, le Géoportail (ou www.cadastre.gouv.fr [communes vectorisées], ou encore google-earth) => cf. 2

Dans tous les cas, on commence ainsi :

Clic sur « Raster » dans le menu et sur « Géoréférencer » dans le menu déroulant qui s’affiche.

$=>$ Une nouvelle fenêtre s'ouvre.

Clic sur « Fichier » dans le menu de cette nouvelle fenêtre, puis sur « Ouvrir un raster » dans le menu déroulant qui s’ouvre, ou bien clic direct sur l’icône .

=> Une fenêtre s'ouvre pour permettre de retrouver le fichier Tif contenant l'image de la carte ancienne. On sélectionne le fichier Tif correspondant à l’image orientée et détourée préparée antérieurement, puis clic sur « Ouvrir ».

=> Une fenêtre « Sélectionneur de Système de Coordonnées de Référence » s’ouvre.

Si « Lambert 93 IGNF:LAMB93 » apparaît dans « Systèmes de Coordonnées de Références récemment utilisés », clic.

Sinon, retrouvez « Lambert93 IGNF :LAMB93 » dans la « Liste des Systèmes de Coordonnées de Référence » disponible en dessous. (on peut aussi taper « epsg:2154 » dans la fenêtre « filtre »).

=> L'image Tif de la carte ancienne dans la fenêtre de géoréférencement qui s’appelle maintenant " Géoréférencer - nom du fichier image.tif » : le plan apparaît sur fond noir.

Clic sur « Paramètres » dans le menu et sélection de « Propriétés du raster » dans le menu déroulant.

=> Une fenêtre « Propriétés des couches - Raster » s'ouvre.

Dans l'onglet « Transparence », clic sur le menu déroulant qui suit « Bande de transparence », sélection de « Bande 4 ». Clic sur OK.

$=>$ Le plan apparaît maintenant sur fond blanc.

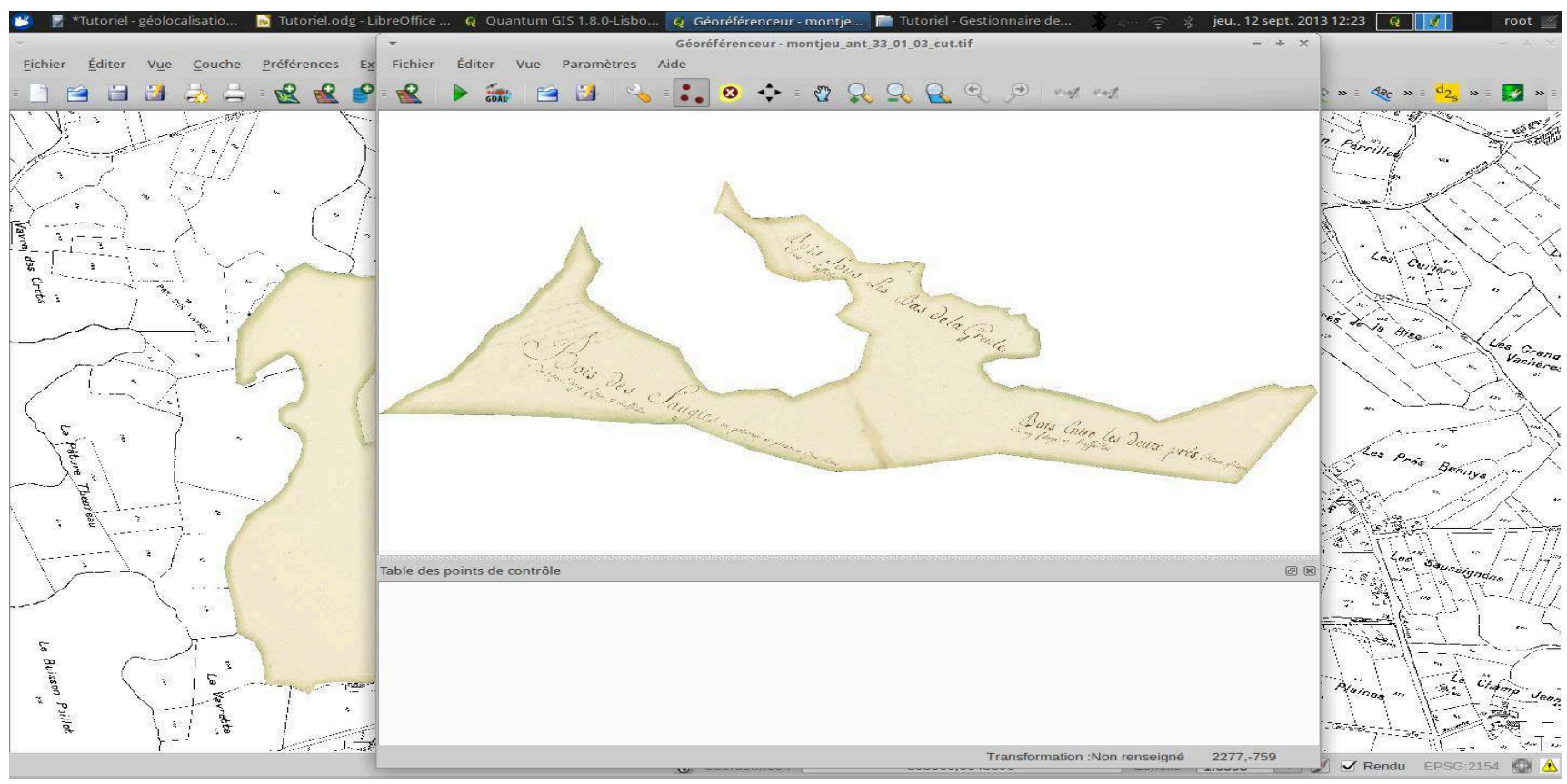




\section{Géolocaliser à partir d'une carte géoréférencée}

Adapter la taille de la fenêtre de géoréférencement afin de pouvoir naviguer facilement entre cette dernière et celle où se trouvent les données géoréférencées (cadastrales et vectorielles), ne pas hésiter à agrandir celle-ci en fermant le panneau des couches vecteur situé à gauche).

En s'aidant des zooms positif et négatif et de la main de déplacement, repérer l'espace couvert par la carte ancienne sur la carte actuelle.

Dans la fenêtre de geéoréférencement, clic sur « Editer » dans le menu et « Ajouter un point » dans le menu déroulant ou sur l'icône

Positionner le curseur sur un point de la carte ancienne et clic gauche.

=> Une fenêtre « Saisir les coordonnées de la carte » s’affiche.

Clic sur « Depuis le canevas de la carte » puis dans l'autre fenêtre, sur le point de la carte actuelle correspondant à celui qui a été sélectionné sur la carte ancienne. La fenêtre se raffiche, clic sur « OK ». $=>$ On a maintenant deux points rouges, un sur chacune des cartes.

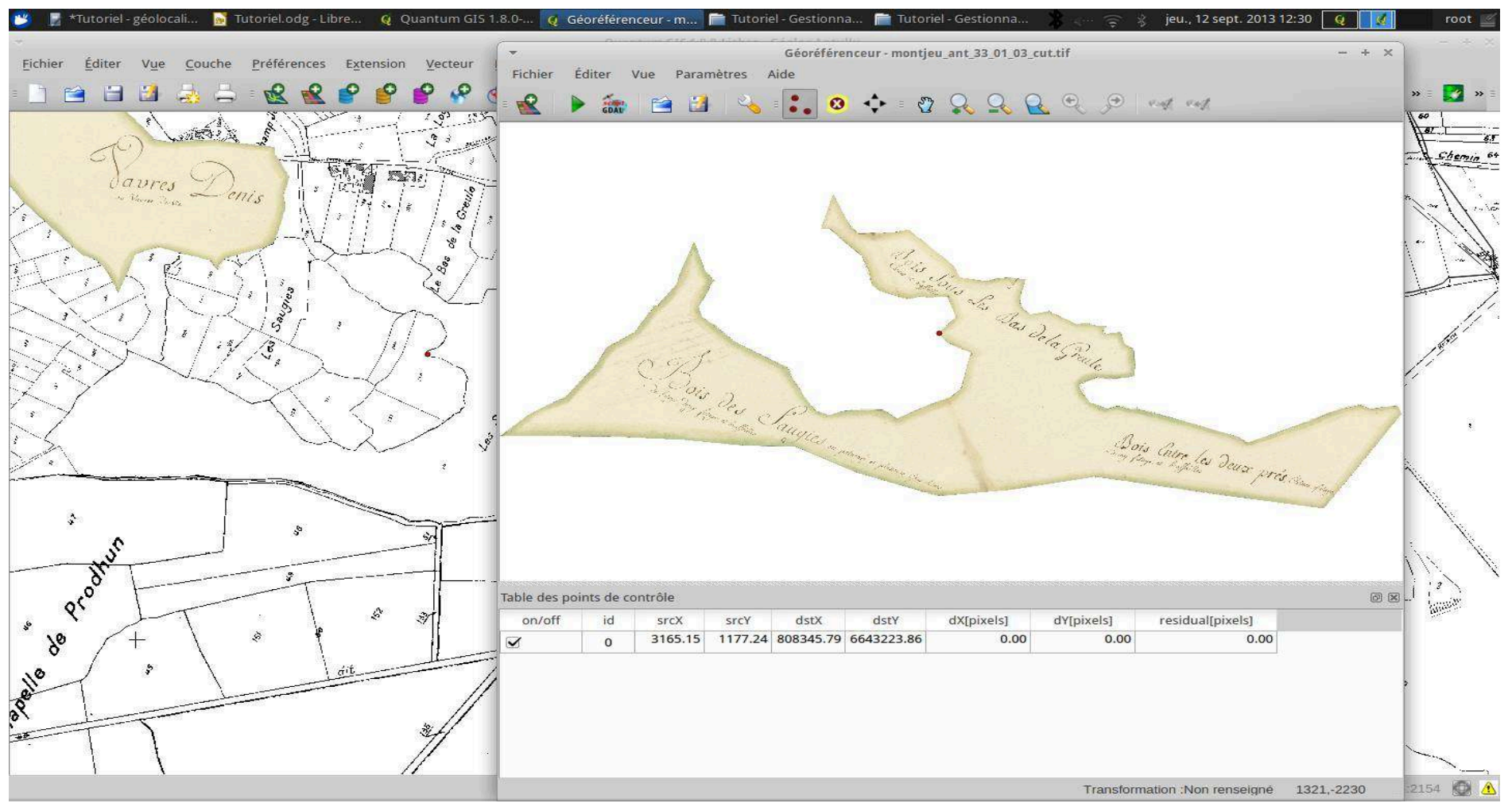

\section{Géolocaliser à partir d'une carte en ligne (www.geoportail.fr ou www.cadastre.gouv.fr)}

Adapter la taille de la fenêtre de géoréférencement afin de pouvoir naviguer facilement entre cette dernière et celle de Géoportail ou de cadastre.gouv.fr.

En s'aidant des zooms positif et négatif et de la main de déplacement, repérer l'espace couvert par la carte ancienne sur la carte actuelle. 
Dans la fenêtre de géoréférencement, clic sur « Editer » dans le menu et « Ajouter un point » dans le menu déroulant (ou sur l’icône ). Positionner le curseur sur un point de la carte ancienne et clic gauche.

=> Une fenêtre « Saisir les coordonnées de la carte » s’affiche.

Repérer les coordonnées du point correspondant sur la carte actuelle et reporter manuellement celles-ci dans la petite fenêtre : on doit avoir une donnée X et une donnée Y. Clic sur « OK ».

\section{3. procédure commune}

Si l'on veut enlever un point, clic sur « Editer » dans le menu puis sur « Enlever un point » (ou sur l'icône enfin sur le point.

Même procédure si l'on veut déplacer le point en question (icône

Placer ainsi au moins vingt-cinq points. S'efforcer de les répartir sur l'ensemble de la carte de manière homogène sans oublier les bords. Pour visualiser le nombre de points et leurs coordonnées, clic sur « Vue » dans le menu et sur « Panneaux » et « Table GCP » dans le menu déroulant.

Enregistrer régulièrement le projet, en cliquant sur « Fichier » dans le menu puis sur «Enregistrez le projet sous » (ou sur l’icône

Si un problème survient et que Qgis se ferme de manière impromptue, le rouvrir, rappeler « projet récent ", et chosir le nom correspondant à la couche de géoréférencemnt ; rouvrir la fenêtre de géoréférencement, du fichier Tif contenant la carte ancienne puis clic sur " Fichier » et « Charger les points de contrôle » dans le menu déroulant (ou sur l'icône

Une fois tous les points nécessaires placés, clic sur « Fichier » et sur « Commencer le géoréférencement » ou bien sur l'icône

=> Une petite fenêtre s’affiche pour demander de préciser le type de transformation.

Clic sur OK.

=> Une fenêtre « Paramètres de transformation » s'ouvre.

Préciser les informations suivantes :

Type de transformation : Thin Plate Spline

Méthode de ré-échantillonnage : Cubic spline

Compression : « Aucune »

Raster de sortie : nom du fichier géoréférencé.tif (n’oubliez pas de préciser le format tif)

SCR Cible : IGNF :LAMB93

Clic sur “OK”, le géoréférencement commence et l'on peut en suivre la progression dans une petite fenêtre.

Une fois le géoréférencement terminé, revenir sur la fenêtre comportant les données de référence et clic sur « Couche » dans le menu, puis sur « Ajouter une couche raster ».

=> La carte ancienne doit s’afficher exactement au dessus de la carte actuelle.

Si elle s'affiche sur fond noir, clic droit sur la couche qui lui correspond dans la table, sélection « Propriétés » dans le menu déroulant qui apparaît. Dans l’onglet « Transparence », sélection comme précédemment de la « Bande 4 ». 


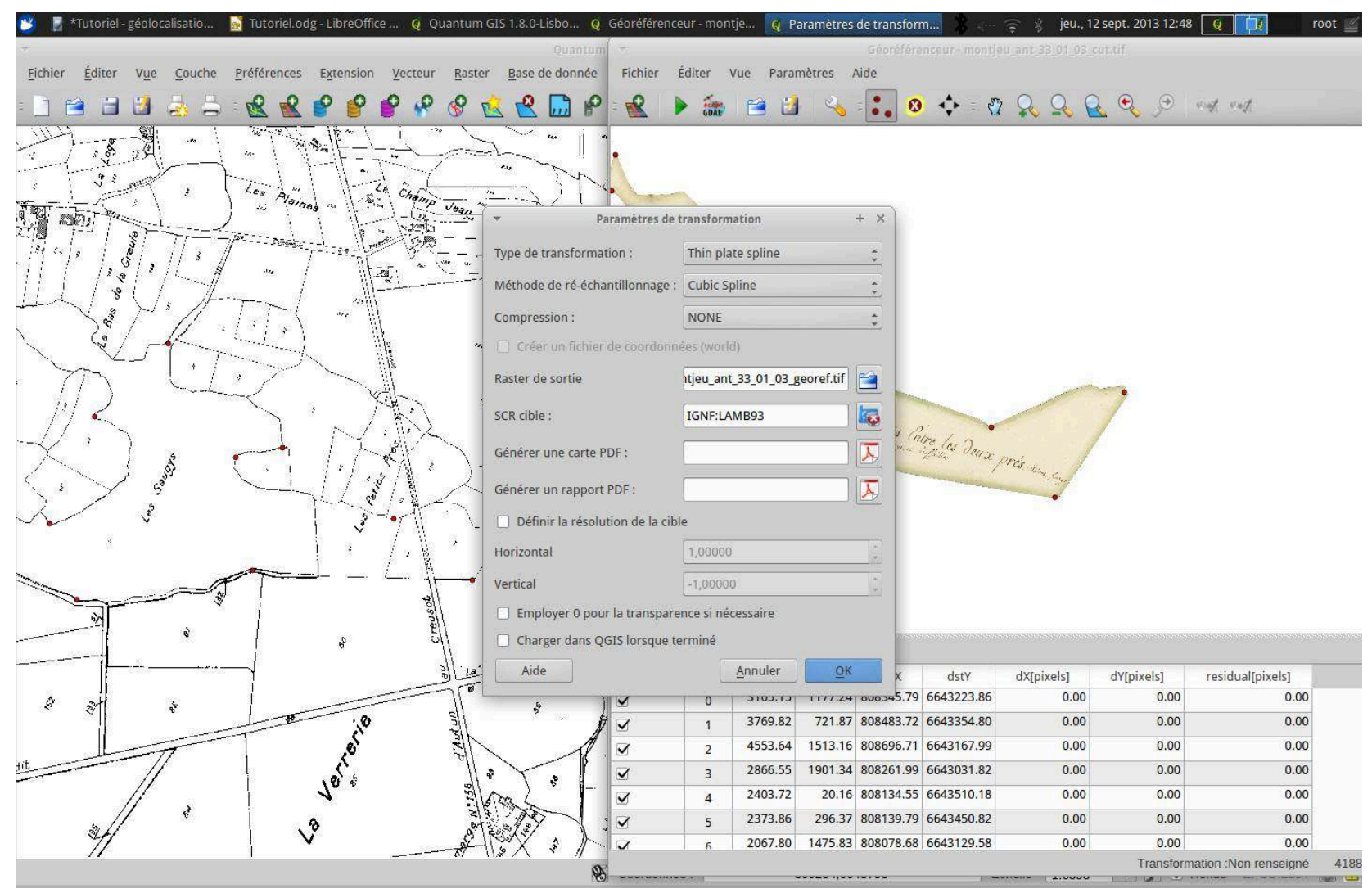

L'opération est terminée !!

\section{RÉSUMÉ}

1. identifier exactement l'endroit représenté.

2. se procurer une carte géoréférencée correspondant à cette zone.

3. en utilisant Gimp, exporter le fichier en TIF,

4. l'orienter si nécessaire,

5. le détourer.

6. en utilisant QGIS, ouvrir la carte de géoréférencement,

7. ouvrir la carte à géolocaliser dans le « georeferencer »,

8. faire correspondre un nombre suffisant de points,

9. lancer la procédure finale,

10. vérifier que la carte traitée s'affiche correctement au dessus de la carte de référence. 
La plupart du temps, les procédures de géoréférencement s'effectuent en reportant sur la carte non géolocalisée les coordonnées de points identiques apparaissant dans des cartes déjà géolocalisées. La difficulté pratique consiste donc à trouver des cartes géolocalisées couvrant la zone à traiter (ci-dessus, II.2).

Il existe à présent une possibilité commode : les 'plugin' OpenLayers et QuickMapServer, qui s'installent facilement dans QGIS et qui permetent d'afficher une grande variété de cartes et de photos aériennes géolocalisées disponibles en ligne sur internet. Toutes les coordonnées sont fournies dans le système de référence epsg:3857, 'wgs84 web pseudo-mercator', qui est le système de géoréférencement utilisé par OSM, Google, Bing, et d'autres. On peut convertir à la volée en lambert93 (epsg:2154) en utilisant le bouton en bas à droite de l'écran.

Cependant, pour la géolocalisation à grande échelle, le plus efficace est le server WMS du Cadastre, qui permet d'afficher dans QGIS le cadastre géolocalisé de n'importe quelle commune. Pour cela,

Couche $>$ Ajouter une couche $>$ Ajouter une couche WMS $>$ Nouveau

On met le nom que l'on veut, et, pour l'URL, il faut mettre http://inspire.cadastre.gouv.fr/scpc/71063.wms?

[en remplaçant 71063 par le numéro d'INSEE de la commune choisie, ici 71063 = Broye]

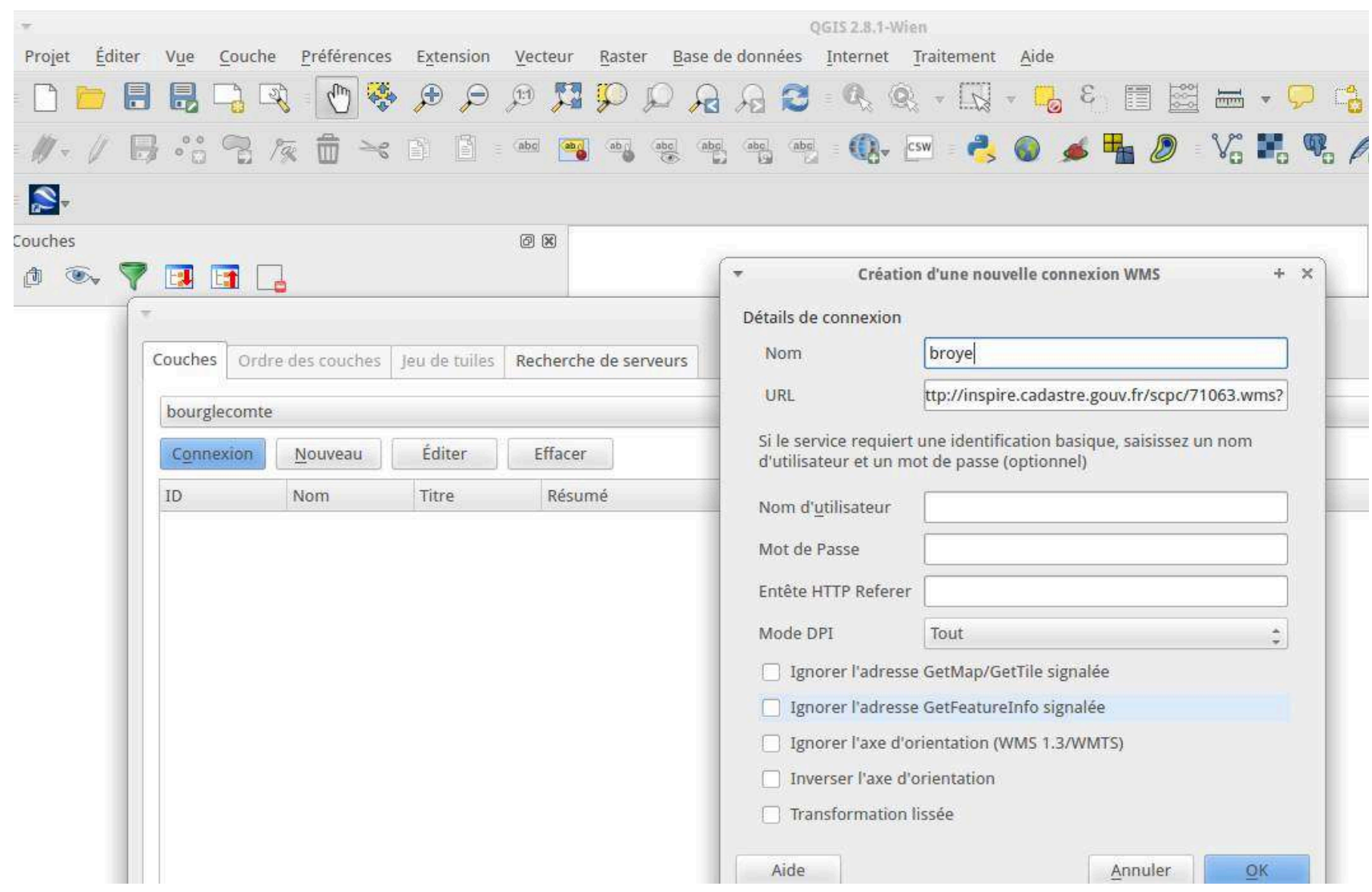

On appuie sur l'onglet connexion, s'affichent toutes les couches disponibles ; on les selectionne toutes, et l'on clique sur 'ajouter' 


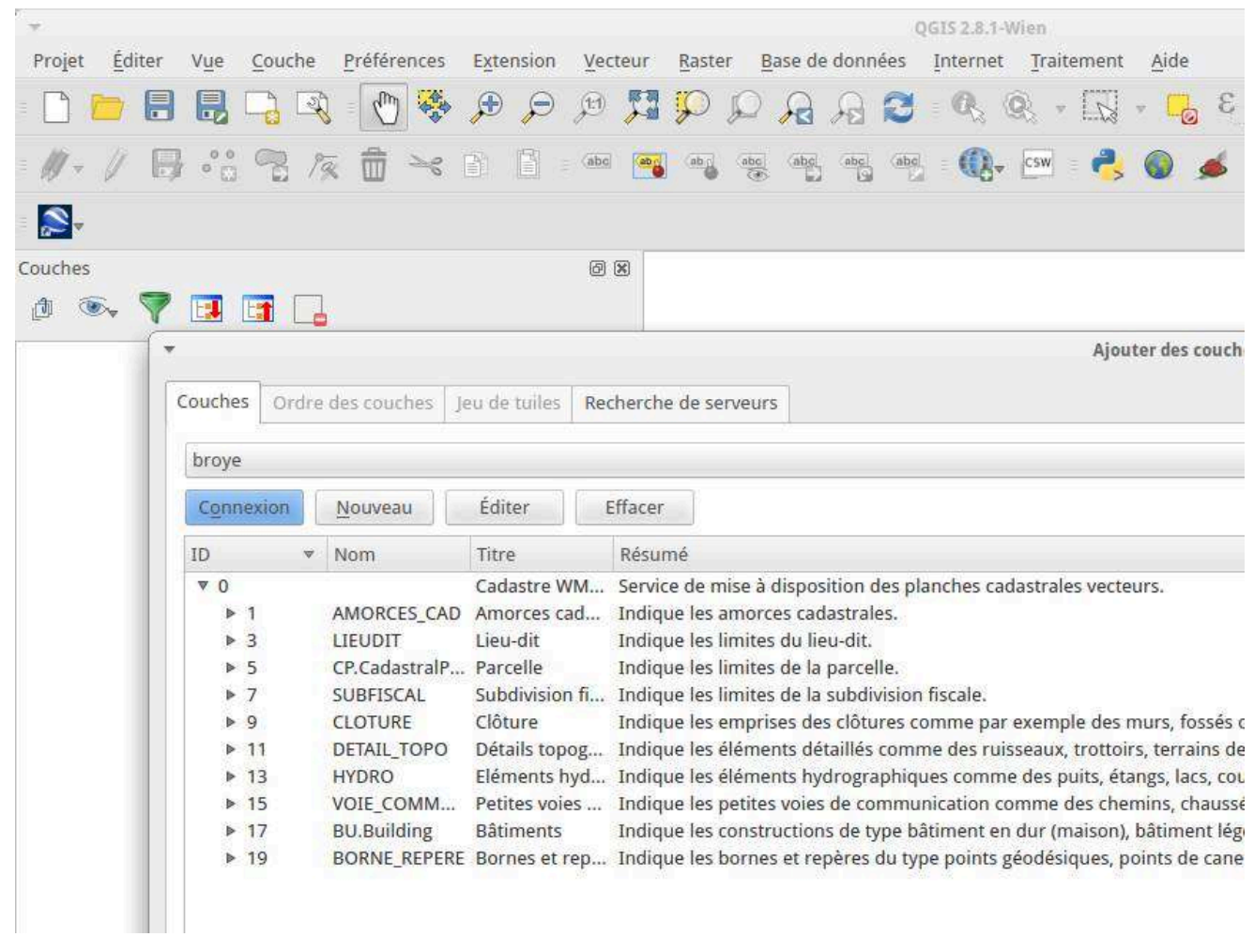

le cadastre entier de la commune s'affiche

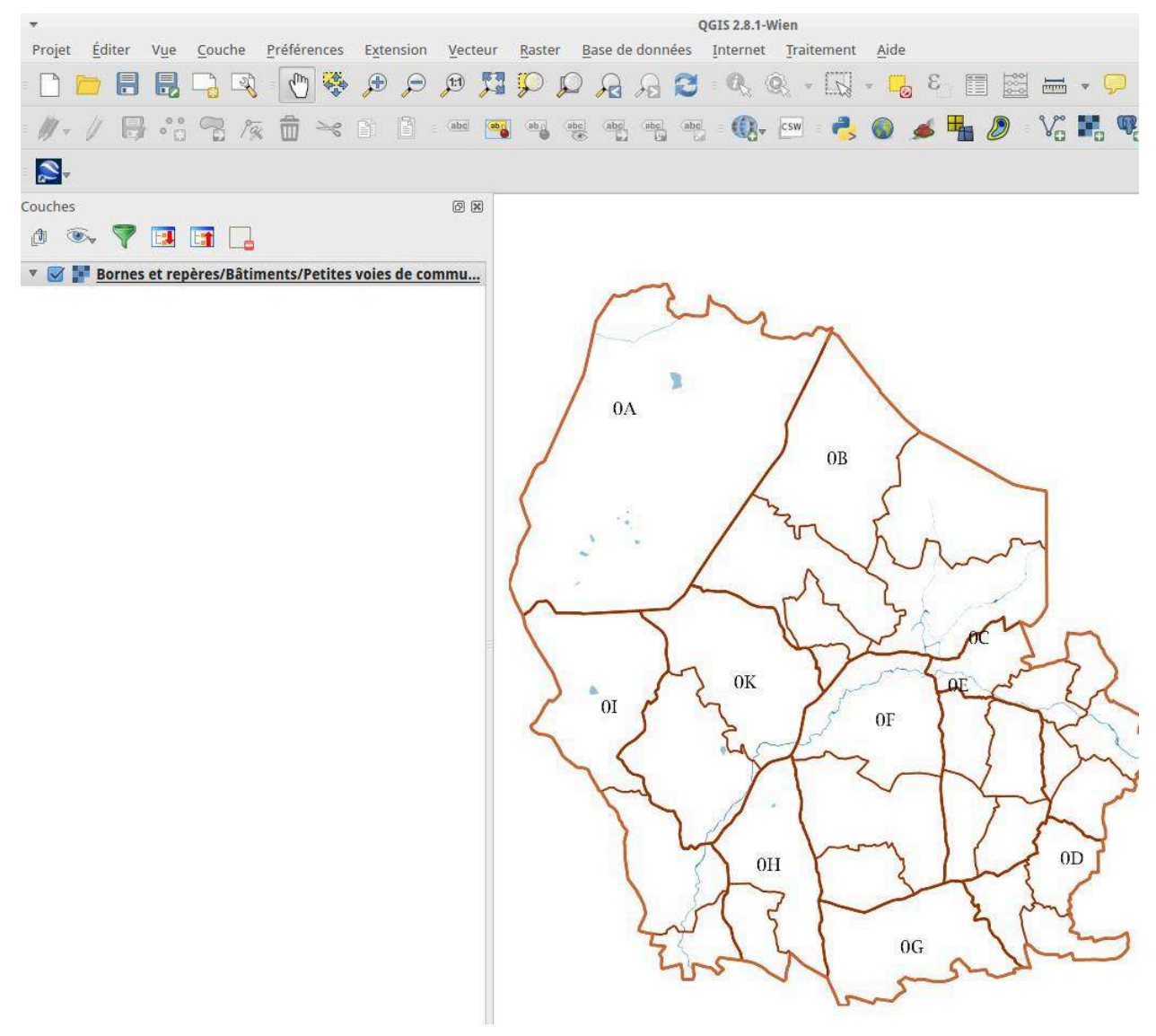

Il suffit de zoomer sur la zone recherchée 


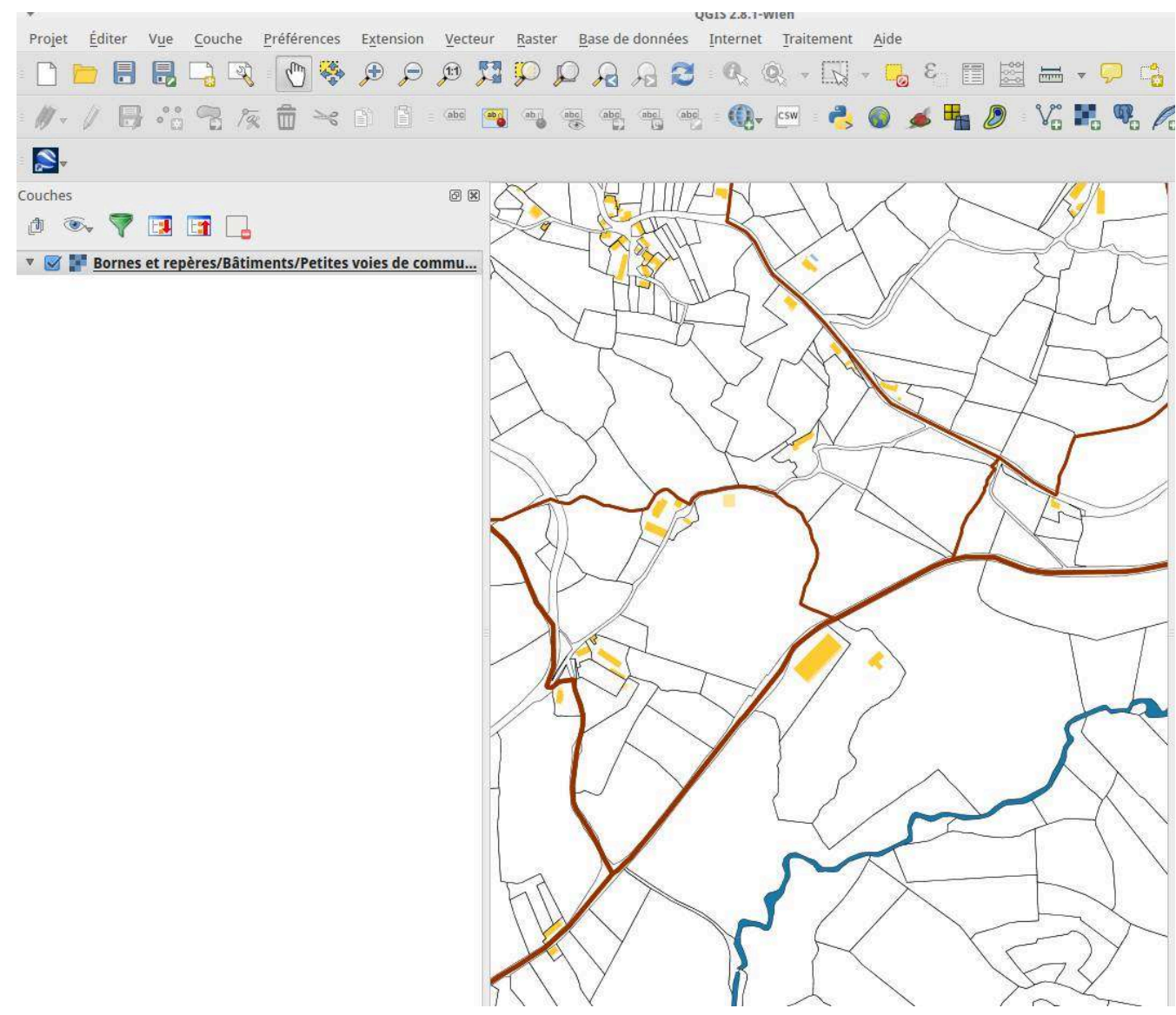

On peut arriver à afficher simultanément une photo aérienne et le cadastre, divers ajustements sont nécessaires (problème des epsg différents)

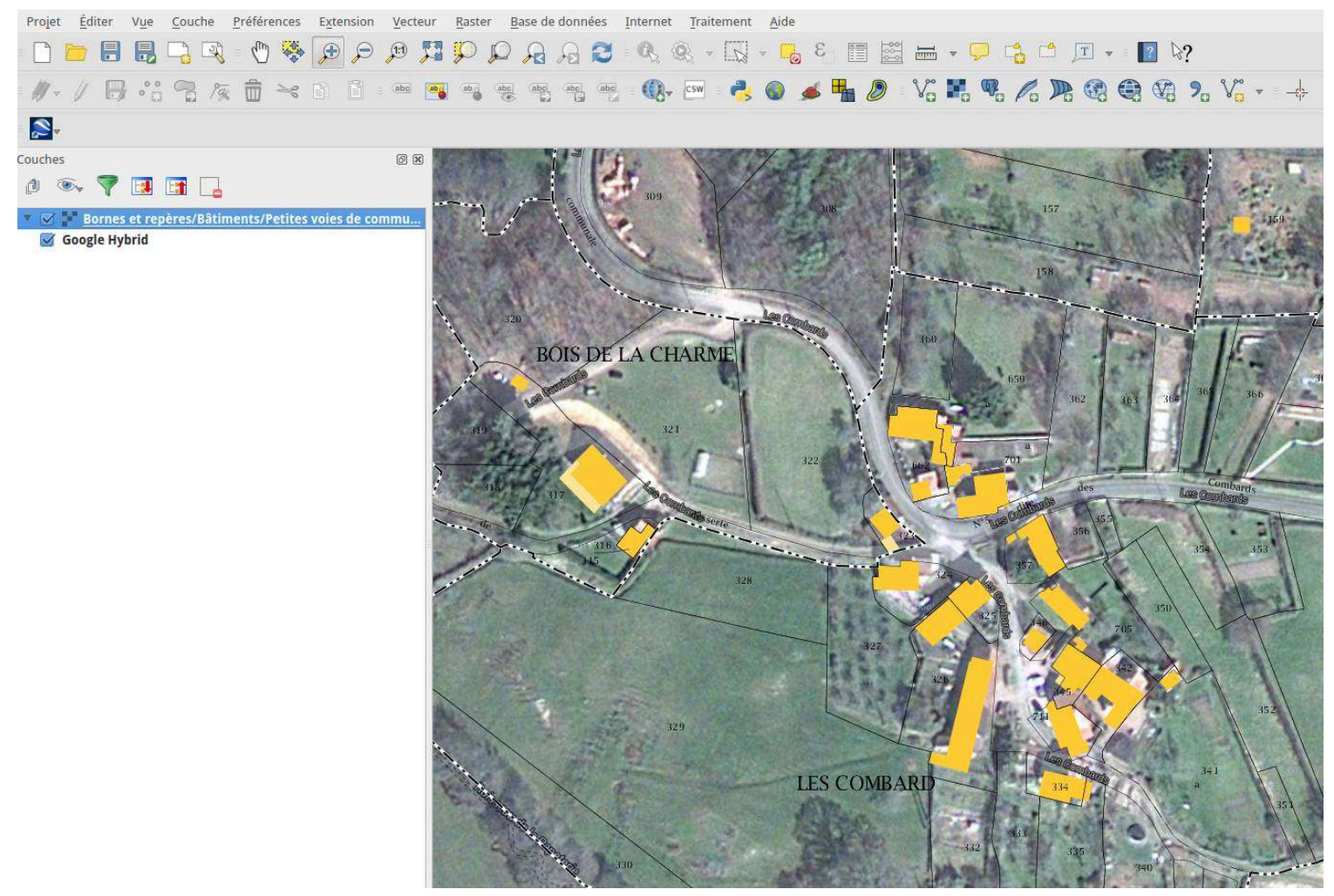


Si l'on travaille en epsg:3857 et que l'on veuille transformer le fichier géolocalisé avec epsg:3857 en epsg:2154 (Lambert93), le plus simple est de le faire en ligne de commande :

gdalwarp -s_srs epsg:3857 -t_srs epsg:2154 input.tif output.tif 